\title{
CONSIDERACIONES ÉTICAS DEL USO DE LA ULTRASONOGRAFÍA 11-14 SEMANAS COMO TAMIZAJE DE ANEUPLOIDÍAS EN LA POBLACIÓN CHILENA
}

\author{
Cristóbal Besio H. ${ }^{1}$, Mauricio Besio R. ${ }^{2}$ \\ 1 Servicio de Obstetricia y Ginecología, ${ }^{2}$ Departamento de Ginecología y Obstetricia, Centro de Bioética, Facultad de
} Medicina, Pontificia Universidad Católica de Chile.

\section{RESUMEN}

El examen ultrasonográfico entre las 11-14 semanas de gestación para pesquisa de aneuploidías en el feto se está realizando sistemáticamente en muchos centros asistenciales de nuestro país. Si bien la utilidad diagnóstica de este examen está ampliamente validada, surgen dudas sobre sus reales beneficios y los potenciales riesgos de este método, utilizado como tamizaje, en nuestra población. Esta presentación tiene como objetivo hacer una reflexión sobre esos riesgos y beneficios. Se discuten la utilización de este procedimiento sin un adecuado consentimiento informado, y las implicancias que tiene la instauración rutinaria de este método en el control obstétrico de nuestra población, especialmente al considerar las condiciones en las que en muchos centros se realiza este examen.

\section{PALABRAS CLAVE: Trans/ucencia nucal, tamizaje, ética}

\section{SUMMARY}

The ultrasound screening for aneuploidies between the 11 and 14 weeks of gestation is systematically performed in many centers of our country. Although the usefulness of this diagnostic test is well known, concern has been raised regarding its potential risks, when used as a screening method. The aim of this article is to analyze the risk and benefits of this practice in our population, considering the frequent lack of an informed consent and how is this procedure performed in several medical centers in our country.

\section{KEY WORDS: Nuchal trans/ucency thickness, screening, ethic}

\section{INTRODUCCIÓN}

Es absolutamente indiscutible el gran aporte que ha producido en los últimos años la introducción del examen ultrasonográfico de las 11-14 semanas en la obstetricia moderna. Numerosa literatura internacional ha validado el uso de este examen, no sólo como screening o tamizaje de aneuploidías, sino además como método de evaluación de la anato- mía fetal (1). Existe evidencia de que el aumento de la translucencia nucal (TN) fetal, además de permitir el cálculo de riesgo de aneuploidías, permitiría detectar fetos con mayor riesgo de presentar cardiopatías, otras anomalías estructurales, síndrome de transfusión feto-fetal en embarazos gemelares monocoriales, y un gran número de diversos síndromes genéticos $(2,3)$. Además este examen permitiría detectar embarazos que tendrían alto riesgo 
de presentar patología placentaria (preeclampsia severa, RCIU) con la utilización del Doppler de arterias uterinas (4).

Esta técnica ha tenido bastante aceptación en la práctica obstétrica de nuestro país, donde en muchos centros incluso ha pasado a ser un examen rutinario en el control obstétrico. Sin embargo, este hecho también ha generado cierta inquietud en algunos especialistas, principalmente debido a que el objetivo por el que fue desarrollado este método diagnóstico, que es la interrupción precoz de un embarazo que presenta una anormalidad, no es aplicable en Chile (5). Por otra parte, la realización de este método de tamizaje en forma rutinaria por personal no capacitado en esta técnica, y la inadecuada entrega de información, muchas veces crea angustia innecesaria en las pacientes, lo que muchos especialistas hemos advertido en nuestra práctica clínica.

Estos hechos nos crean las siguientes interrogantes, sobre las cuales parece necesaria alguna reflexión: ¿es necesario el cálculo de riesgo de aneuploidía en todas las pacientes, como método de tamizaje?, y si es así ¿se está realizando bajo las condiciones adecuadas y establecidas para ello?

\section{Cálculo de riesgo para aneuploidías}

El examen ultrasonográfico a las 11-14 semanas permite calcular el riesgo individual de presentar no sólo trisomía 21 , sino además trisomías 13, 18 y síndrome de Turner (6). Este cálculo se realiza al incorporar al riesgo basal de la paciente (edad materna, edad gestacional al momento del examen, antecedente presente o ausente de aneuploidía en un embarazo previo), la medición de la TN, ya que al aumentar ésta, aumenta el riesgo de aneuploidía. Esta información permite predecir el riesgo individual de presentar trisomía 21 con una sensibilidad de un $72 \%$, con una tasa de falsos positivos de un $5 \%$ (6). Al sumar a la medición de TN, la presencia o ausencia de hueso nasal y los marcadores bioquímicos de primer trimestre (PAPP-A y fracción libre de B-HCG) se aumenta la sensibilidad del examen hasta un $97 \%$, manteniendo la tasa de falsos positivos en $5 \%$ (7).

Este cálculo de riesgo individual permite ofrecer a las pacientes de mayor riesgo la alternativa de ser sometidas a un estudio cromosómico fetal para un diagnóstico definitivo, el cual puede ser realizado mediante una biopsia de vellosidades coriales 0 una amniocentesis, según la edad gestacional. A su vez, permite a las pacientes con bajo riesgo poder rechazar el estudio cromosómico, que no está libre de complicaciones. Esto último especialmente, en países donde está permitido el aborto eugenésico.

Es muy importante destacar que estos buenos resultados, en cuanto a la sensibilidad de este examen, fueron obtenidos principalmente por el grupo del King's College Hospital en Londres, que cuenta con especialistas con mucha experiencia en esta técnica, ya que es innegable que la medición correcta de la TN es una técnica difícil, operador dependiente, que requiere experiencia y que debe cumplir con una estandarización rigurosa definida por la Fetal Medicine Fundation (1) (Tabla I).

Tabla I

\section{TÉCNICA DE MEDICIÓN DEL MÁXIMO GROSOR DE LA ZONA ANECOGÉNICA SUBCUTÁNEA UBICADA ENTRE LA PIEL Y LAS PARTES BLANDAS QUE RECUBREN LA ESPINA CERVICAL DEL FETO}

\footnotetext{
- Entre 11-13+6 semanas, con longitud céfalo nalgas entre 45 y $84 \mathrm{~mm}$.

- Corte sagital medio del feto, con éste en posición neutra.

- Magnificación adecuada. La cabeza fetal y el tórax superior deben ocupar el $75 \%$ de imagen.
}

- Cálipers con graduación 0,1mm.

- Piel separada del amnios.

- Cálipers situados en los bordes internos de líneas que definen TN.

- Considerar la mayor de 3 mediciones.

\section{Ecografía 11-14 semanas como prueba de screening}

Se entiende como prueba de screening o tamizaje, un examen que se aplica a una población asintomática con el fin de determinar aquellas personas susceptibles de tener la patología que es objeto del tamizaje, para que éstas puedan ser investigadas hasta su diagnóstico final (8). Una prueba de tamizaje debe tener como características ser un examen fácil de realizar, aplicable a una población, con buena relación costo-beneficio, que su aplicación no genere efectos adversos sobre la salud, que existan pruebas confirmatorias para un diagnóstico definitivo, y quizás lo más importante, ofrecer un tratamiento adecuado y oportuno para la patología investigada. El Papanicolaou y la mamografía son 
claros ejemplos de exámenes de tamizaje que han demostrado reducir la mortalidad de cáncer de cuello uterino y de mama, respectivamente.

Existen muchos países donde está permitido legalmente la interrupción precoz de embarazos con anormalidades. En estas sociedades no existen mayores cuestionamientos éticos sobre esta acción y, más aún, los individuos con cromosomopatías son considerados como una carga social y como un "costo económico" innecesario para el Estado. Es muy importante tener en cuenta que el objetivo primordial por el cual fue desarrollada la ecografía 11-14 semanas, en estos países, es la detección de fetos con aneuploidías y/o con anormalidades estructurales para poder abortarlos precozmente, teniendo en cuenta que la no detección de estas anormalidades tiene, además, implicancias médico-legales. En este contexto, la ecografía 11-14 semanas como método de screening a toda la población obstétrica permite seleccionar a las pacientes que irán a un estudio cromosómico definitivo para así interrumpir los embarazos con anormalidades en forma precoz. Asimismo, este examen permite tranquilizar a las pacientes catalogadas de bajo riesgo y darles la opción de no ser sometidas a un estudio invasivo definitivo.

En nuestro país, así como en muchos otros países de nuestro continente, existe una realidad distinta. El aborto eugenésico no solo está penalizado por la ley (5), sino que además, un porcentaje importante de nuestra población condena esta práctica por razones éticas, culturales y religiosas. En este contexto es necesario preguntarse ¿qué realmente le estaríamos ofreciendo como beneficio a la población obstétrica con este examen de tamizaje?

Es muy válido plantear que una ecografía 11-14 semanas que resulta dentro de límites normales es un hecho que resulta muy tranquilizador en el embarazo de una madre. También es planteable que, basado sólo en el principio de la autonomía de la madre, una paciente tendría el derecho de conocer el riesgo que tiene su hijo de presentar una anomalía y tener la alternativa de solicitar un diagnóstico definitivo mediante un examen invasivo. Sin embargo, ¿justifican estos hechos la realización de este procedimiento como tamizaje rutinario a toda la población obstétrica, siendo que en Chile no existe la opción eugenésica, ni tampoco alguna acción terapéutica para el feto? (5), ¿no sería más válida la alternativa de ofrecer a las pacientes este examen, y que ellas puedan decidir libremente su realización, luego de una información adecuada?

Es inquietante para algunos especialistas el hecho de que, en cada vez más centros ecográficos, se realice en forma rutinaria la medición de la
TN y la visualización del hueso nasal a las 11-14 semanas, sin la autorización previa de la paciente y sin la adecuada entrega de información. Es más inquietante aún, el que en muchos de estos centros el personal que realiza este examen no tiene la suficiente experiencia para realizarlo de manera adecuada. Todo esto tiene como consecuencia, muchas veces, el provocar una angustia innecesaria en las pacientes, y en otras, la no detección de un feto con riesgo aumentado de presentar una aneuploidía. Estos hechos advertidos en nuestra práctica clínica, se producen al querer instaurar la ecografía 11-14 semanas como un examen básico de rutina en el control del embarazo.

\section{Otras consideraciones de la ecografía 11-14 semanas}

Además del cálculo de riesgo para aneuploidías, el examen ultrasonográfico entre las 11-14 semanas permite una evaluación inicial de la anatomía fetal. Está ampliamente descrito en la literatura que durante esta edad gestacional es posible diagnosticar algunas anormalidades estructurales mayores del feto. No hay lugar a dudas de que una evaluación inicial normal de la anatomía fetal, sumada al hecho de que a esta edad gestacional existe estadísticamente un descenso brusco del riesgo de presentar un aborto espontáneo, genera tranquilidad en una paciente embarazada. Sin embargo, siempre hay que tener en cuenta que el examen "gold standard" para evaluar la anatomía fetal es una ecografía a las 22-24 semanas, examen fundamental en el control obstétrico, que permite una visualización mucho mejor de las estructuras fetales, y frente al hallazgo de alguna anormalidad permite un mejor diagnóstico, entregar un pronóstico, derivar a estas pacientes a centros de mayor complejidad para un manejo neonatal adecuado, y en algunos casos permite ofrecer terapia fetal. No hay que desconocer que el análisis minucioso de la anatomía fetal a las 11-14 semanas tiene como objetivo primordial la interrupción precoz de embarazos con anormalidades en países donde el aborto es permitido. Pese a que es válido informar y ofrecer este examen a nuestras pacientes para una evaluación inicial de la anatomía fetal, ¿será necesaria la ecografía 11-14 semanas para evaluar anatomía fetal como control rutinario del embarazo en nuestro medio, sabiendo que igualmente todas nuestras pacientes deberían realizarse una ecografía entre las 22-24 semanas?

Por último, es sabido que una TN aumentada, además de incrementar el riesgo de presentar aneuploidías, aumenta el riesgo de presentar malformaciones estructurales, especialmente cardiopatías, y también aumenta el riesgo de presentar 
un síndrome de trasfusión feto-fetal en embarazos gemelares monocoriales. Sin embargo, como ya hemos visto, en el caso de las cardiopatías, la mejor evaluación del corazón fetal se obtiene con la ecografía 22-24 semanas, siendo éste el examen que debería determinar una eventual derivación a un centro de mayor complejidad para una ecocardiografía fetal. En el caso de los embarazos gemelares, el diagnóstico de corionicidad se debería determinar en la ecografía de primer trimestre, y todos los embarazos gemelares monocoriales deben tener un control ecográfico seriado a partir del segundo trimestre para el diagnóstico precoz del síndrome de transfusión feto-fetal, independientemente de la medición de la TN.

\section{DISCUSIÓN}

El examen ultrasonográfico de las 11-14 semanas cumple varias funciones, siendo la más importante el cálculo de riesgo de presentar una aneuploidía, principalmente la trisomía 21. Este hecho ha creado gran entusiasmo en nuestro país en los últimos años, en donde cada vez más se ocupa esta herramienta diagnóstica como examen rutinario en el control del embarazo. Pese a que la validez de este examen no merece cuestionamiento alguno, sí es importante preguntarse cuál es la utilidad de éste como método de tamizaje para aneuploidías en nuestra población obstétrica. Recordemos que no existen en la actualidad intervenciones terapéuticas para esta condición, y la opción eugenésica se descarta por razones legales en Chile (5), teniendo además importantes reparos éticos. Claramente el hecho de tener un examen ultrasonográfico entre las 11-14 semanas dentro de la normalidad genera tranquilidad en una paciente embarazada. También es planteable el hecho de que un examen alterado, con una confirmación diagnóstica invasiva posterior de aneuploidía, pudiera permitir una preparación psicológica para afrontar de mejor manera esta situación. Sin embargo, basándose en el respeto de la autonomía de la madre, también una paciente tiene todo el derecho de no querer saber cuál es su riesgo. En estos casos se estaría vulnerando este derecho, al realizar la medición de la TN en forma sistematizada a toda la población, sin una información adecuada.

Otro punto que hay que considerar es que al querer implementar este examen como tamizaje masivo, debido al interés de ofrecer toda la tecnología actual a las pacientes, se corre el riesgo de que, en muchos centros de salud el personal que realiza este examen no esté debidamente certificado o no tenga experiencia suficiente en esta técnica. Este hecho tiene efectos negativos, ya que puede gene- rar angustia y preocupación, innecesariamente, en muchas pacientes.

La medición de la TN, podría ser considerada como método de screening para otras anormalidades, especialmente para las cardiopatías, ya que nos permitiría seleccionar qué pacientes deberían ser referidas a centros terciarios para un estudio ecocardiográfico fetal. Sin embargo, como ya hemos visto, nuestros mejores esfuerzos deberían estar orientados a que la población obstétrica tenga acceso a una ecografía 22-24 semanas de calidad, ya que éste es el mejor examen de screening para detectar este tipo de anormalidades.

La ecografía 11-14 semanas, como tamizaje de aneuploidías, se debería ofrecer a todas las pacientes con una información adecuada, para que éstas puedan elegir libremente si desean acceder a la información que este examen pueda entregar. Además, es fundamental que el personal que lo realice tenga experiencia en esta técnica, siendo lo ideal el que estén certificados mediante el curso que ofrece la Fetal Medicine Fundation para la correcta medición de la TN.

\section{CONCLUSIÓN}

En Chile la ecografía 11-14 semanas como tamizaje de aneuploidías se debería ofrecer a todas las pacientes, siempre y cuando sea con una adecuada información para que cada paciente pueda elegir libremente si desea acceder a este examen. Esto debido a que pueden existir pacientes que no desean conocer cual es su riesgo, y a que actualmente no existen intervenciones terapéuticas para fetos con aneuploidías ni existe la opción de un aborto eugenésico. Todas las pacientes que decidan someterse a este examen deberían tener la alternativa de realizarlo con un especialista con experiencia en esta técnica, ojalá certificados por el curso que ofrece la Fetal Medicine Fundation, para así evitar los efectos adversos que se producen al instaurar esta técnica en forma sistematizada, como ocurre actualmente en Chile en muchos centros ultrasonográficos.

\section{BIBLIOGRAFÍA}

1. Dezerega V, Sepúlveda W. El examen ultrasonográfico de las 11-14 semanas. En: Guzmán E, Rodríguez N, Ruiz M (Eds). Selección de Temas en Ginecoobstetricia, $1^{\underline{a}}$ ed. Santiago: Ediciones Publimpacto, 2005;47-65.

2. Souka A, Snijders R, Novakov A, Soares W, Nicolaides K. Defects and syndromes in chromosomally normal fetuses with increased nuchal translucency thickness at 10-14 weeks of gestation. Ultrasound Obstet Gynecol 1998;11:391-400. 
3. Sebire N, D' Ercole C, Hughes K, Carvalho M, Nicolaides $\mathrm{K}$. Increased nuchal translucency thickness at 10-14 weeks of gestation as a predictor of severe twin-to-twin transfusion syndrome. Ultrasound Obstet Gynecol 1997;17:467-70.

4. Parra M, Rodrigo R, Barja P, Bosco C, Fernández V, Muñoz H, Soto-Chacón E. Screening test for preeclampsia through assessment of uteroplacental blood flow and biochemical markers of oxidative stress and endothelial dysfunction. Am J Obstet Gynecol 2005;193(4):1486-91.

5. Códico Sanitario de la República de Chile. Artículo 119. Editorial Jurídica de Chile, Edición Oficial, Aprobado por Decreto № 418, de 18 de mayo de 2004, del Ministerio de Justicia. Santiago, Chile.
6. Snijders R, Noble P, Sebire N, Souka A, Nicolaides $\mathrm{K}$. UK multicentre project on assessment of risk of trisomy 21 by maternal age and fetal nuchal translucency thickness al 10-14 weeks of gestation. Fetal Medicine Foundation First Trimester Screening Group. Lancet 1998:352:343-6.

7. Cicero S, Curcio P, Papageorghiou A, Sonek J, Nicolaides $\mathrm{K}$. Absence of nasal bone in fetuses with trisomy 21 at 11-14 weeks of gestation: An observational study. Lancet 2001;358:1665-7.

8. Salinas $H$, Valdés $E$, Carmona $S$. Screening genético antenatal para la detección de aneuploidías. Rev Chil Obstet Ginecol 2003;68(6):529-35. 